\title{
Weight cycling and the risk of type 2 diabetes in the EPIC-Germany cohort
}

\author{
Jasmine Neamat-Allah $^{1}$ • Myrto Barrdahl ${ }^{1}$ - Anika Hüsing ${ }^{1} \cdot$ Verena A. Katzke $^{1}$. \\ Ursula Bachlechner ${ }^{2}$ - Annika Steffen ${ }^{2}$ - Rudolf Kaaks ${ }^{1}$ - Matthias B. Schulze ${ }^{3,4}$. \\ Heiner Boeing ${ }^{2}$ - Tilman Kühn ${ }^{1}$
}

Received: 18 February 2015 / Accepted: 20 August 2015 / Published online: 16 September 2015

(C) Springer-Verlag Berlin Heidelberg 2015

\begin{abstract}
Aims/hypothesis Studies on weight cycling and the risk of type 2 diabetes have revealed inconsistent results, possibly due to differences in the definition of weight fluctuations. Here, we investigated whether weight cycling during adulthood is related to diabetes risk in a large cohort study, using a complementary approach to define patterns of weight development.

Methods Weight cycling, weight loss and weight gain were defined (1) a priori, using distinct categories, and (2) by functional principal component analysis (FPCA) to capture weight patterns in greater detail. Associations of weight cycling, weight loss and weight gain with the risk of type 2 diabetes were evaluated by Cox regression models.

Results A priori defined weight cycling was associated with increased diabetes risk, compared with stable weight (HR 1.36 [95\% CI 1.09, 1.68]). No significant association between FPCA-derived weight cycling and risk of diabetes was observed after adjustment for concurrent weight patterns (HR
\end{abstract}

Electronic supplementary material The online version of this article (doi:10.1007/s00125-015-3755-9) contains peer-reviewed but unedited supplementary material, which is available to authorised users.

Tilman Kühn

t.kuehn@dkfz.de

1 Division of Cancer Epidemiology, German Cancer Research Center (DKFZ) Heidelberg, Im Neuenheimer Feld 581, D-69120 Heidelberg, Germany

2 Department of Epidemiology, German Institute of Human Nutrition Potsdam-Rehbrücke (DIfE), Nuthetal, Germany

3 Department of Molecular Epidemiology, German Institute of Human Nutrition Potsdam-Rehbruecke (DIfE), Nuthetal, Germany

4 German Center for Diabetes Research (DZD), Neuherberg, Germany
$1.19[95 \%$ CI $0.89,1.60])$. Subgroup analyses showed that FPCA-derived weight cycling during net weight gain was associated with a higher risk of diabetes (HR 1.68 [95\% CI $1.14,2.48]$ ). A priori defined weight gain (HR 2.08 [95\% CI $1.60,2.70])$ was more clearly related to the risk of diabetes than FPCA-derived weight gain (HR 1.20 [95\% CI 0.95, 1.51]), while no significant associations were observed for weight loss.

Conclusions/interpretation Overall, weight cycling may not be an independent risk factor for type 2 diabetes when accounting for concurrent patterns of weight development. However, weight cycling may pose a stronger risk of diabetes than non-cycling during net weight gain.

Keywords Prospective study - Type 2 diabetes · Weight change $\cdot$ Weight cycling

$\begin{array}{ll}\text { Abbreviations } \\ \text { DPP } & \text { Diabetes Prevention Program } \\ \text { EPIC } & \begin{array}{l}\text { European Prospective Investigation into Cancer } \\ \text { and Nutrition }\end{array} \\ \text { FHS } & \text { Framingham Heart Study } \\ \text { FPCA } & \text { Functional Principal Component Analysis } \\ \text { IWS } & \text { Iowa Women's Health Study } \\ \text { NHS II } & \text { Nurses' Health Study II } \\ \text { PC } & \text { Principal component } \\ \text { TOPS } & \text { Taking Off Pounds Sensibly }\end{array}$

\section{Introduction}

While it is well established that overweight, obesity and weight gain are major risk factors for type 2 diabetes [1, 2], the consequences of weight cycling, i.e. repeated cycles of 
intentional weight loss followed by weight regain (or vice versa), are less clear. Only a few large-scale prospective studies on weight cycling and type 2 diabetes risk (with mixed results) have been published to date. Early findings from the Iowa Women's Health Study (IWS) and the Taking Off Pounds Sensibly (TOPS) programme indicated that weight cycling may lead to type 2 diabetes in middle-aged women $[3,4]$. In contrast, no significant associations between weight cycling and type 2 diabetes risk were observed in participants of the Nurses' Health Study (NHS II) and the Framingham Heart Study (FHS) in two subsequent analyses [5, 6]. Both the latter results and null findings from studies on weight cycling and other cardiometabolic endpoints led to the notion that adverse effects of weight cycling might not exist at all [7]. However, in the lifestyle arm of the Diabetes Prevention Program (DPP), it was recently shown that even mild weight cycling was associated with an increased risk of type 2 diabetes, whereas there were no relationships with other cardiometabolic traits [8].

Inconsistent results of these previous studies may be related to differences with respect to the magnitude and frequency of weight changes underlying a priori definitions of weight cycling used [7]. Furthermore, it can be speculated that weight cycling may lead to different outcomes depending on whether or not an individual experiences long-term net weight change. This was hardly taken into consideration in previous studies and cannot be accounted for when using distinct a priori definitions of weight cycling. Interestingly, there has been only one study on weight cycling and type 2 diabetes so far in which concurrent patterns of overall weight status, net weight change and weight cycling were investigated. In the FHS, Waring et al applied functional principal component analyses (FPCA) to characterise patterns of weight change over time [6]. While, in fact, a significant association between FPCAderived weight cycling and the risk of type 2 diabetes was observed in a crude statistical model, this association was no longer significant after adjustment for concurrent FPCAderived patterns of overall weight status and net weight change [6].

The aim of the current study was to investigate the relationship between weight cycling, as classified by (1) a priori defined categories that have been shown to be related to increased risk of type 2 diabetes in previous studies [3, 8], and (2) FPCA-derived categories and the risk of type 2 diabetes. The latter FPCA-based approach was used because (unlike the first) it facilitates simultaneous modelling of concurrent patterns of weight development such as weight cycling and weight gain. We hypothesised that weight cycling (as defined by both approaches) is a risk factor for type 2 diabetes, albeit with the expectation that associations between FPCA-derived weight cycling and the risk of type 2 diabetes would be attenuated when taking concurrent patterns of weight development into account. Thus, we evaluated a priori classified and FPCA- derived patterns of weight cycling in relation to the risk of type 2 diabetes over time in a large-scale cohort study, the German branch of the European Prospective Investigation into Cancer and Nutrition (EPIC) [9].

\section{Methods}

Ethics statement The study was approved by local ethics committees (Potsdam: Ethics Committee of the Medical Association of the State of Brandenburg; Heidelberg: Ethics Committee of the Heidelberg University Medical School) and all participants gave written informed consent.

Study population EPIC-Germany initially comprised 53,088 participants (Heidelberg: $n=25,540$ [53\% women]; Potsdam: $n=27,548$ [60\% women]) aged between 35 and 65 years, and recruited between 1994 and 1998 from the local general populations [9]. At baseline, anthropometric measurements (weight, height, waist and hip circumferences) were taken by trained staff according to standardised protocols. Lifestyle characteristics (e.g. physical activity, diet, alcohol consumption, smoking history), health status and socioeconomic status were assessed by personal interviews and questionnaires. Follow-up information was obtained by self-administered questionnaires which were sent to the participants at average intervals of $2-3$ years.

Re-assessment of weight during follow-up For the present study, self-reported information on weight as recorded during three follow-up waves was used and corrected by validated equations published elsewhere $[10,11]$. Each of the follow-up waves included one re-assessment of weight by questionnaire per individual. In the questionnaire, participants were asked to measure their weight with minimal clothing on the day they filled out the questionnaire, without further specifications. All statistical analyses described here are based on one measured weight value from baseline and up to three self-reported weight values from follow-up, i.e. a maximum of four weight assessments per individual. Details on the assessments of weight during follow-ups of the EPIC cohorts in Heidelberg and Potsdam used for the present study are shown in Fig. 1.

Case ascertainment Incident cases of type 2 diabetes were identified by self-reported disease occurrence and use of specific medication in follow-up questionnaires, record linkage with local clinics and death certificates. All potential cases were verified by study physicians based on medical records provided by treating physicians. Only incident cases, which had occurred after the final weight re-assessment and before 31 December 2009 were included in the present analyses (see below). 


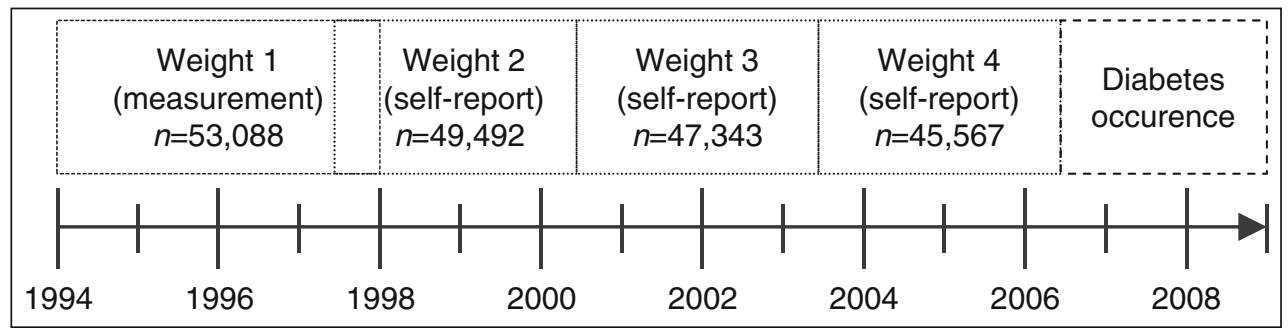

Fig. 1 Schematic representation of repeated weight assessments in the EPIC-Germany cohort over time. For the present analyses, up to four weight assessments overall per study participant were available and the numbers of responders per follow-up are given. The first weight assessment was part of the baseline examination at the study centres and carried out by trained personnel. All three consecutive weight assessments are derived from questionnaires sent to the participants as part of three subsequent follow-ups. The time frames of the baseline examination and the follow-up waves, in which one weight assessment per individual was carried out, are represented by the boxes and the timeline
Exclusions Participants were excluded according to the following criteria, as shown in electronic supplementary material (ESM) Fig. 1: prevalent diabetes $(n=2,512)$; prevalent or incident cancer $(n=1,086)$; prevalent or incident major cardiovascular diseases (myocardial infarction, $n=1,368$; stroke, $n=1,176)$; missing baseline covariates $(n=217)$; and incident diabetes that occurred before the last weight assessment $(n=1,519)$. Major cardiovascular disease patients were excluded to avoid bias due to unintentional weight loss. For analyses on weight change using a priori defined cut-offs, participants with missing information on weight from more than one out of three re-assessments $(n=147)$ were also excluded, resulting in a final dataset of 45,063 participants. For FPCA analyses (see below), only participants with weight information from all three re-assessments during follow up $(n=35,270)$ were included.

A priori defined categories of weight change The average (SD) duration between individual re-assessments of weight during follow-up was 2.54 years ( \pm 0.83 years) in Heidelberg, 2.23 years $( \pm 0.46$ years $)$ in Potsdam and 2.39 years ( \pm 0.67 years) overall. Weight cycling was first defined by average annual weight loss of $\geq 1.125 \mathrm{~kg}$ between two consecutive weight assessments and subsequent regain (or vice versa). This definition was based on a previous report by the DPP in which a weight shift of $\geq 2.25 \mathrm{~kg}$ over a 2 year period (i.e. $1.125 \mathrm{~kg} /$ year) and a subsequent reverse shift of the same magnitude was considered a weight cycle [8]. As the duration of individual follow-up waves differed slightly between participants of the EPICGermany study in Heidelberg and Potsdam (see above), a $1.125 \mathrm{~kg} /$ year threshold was applied in the present study. In addition, annual average changes of 0.75 and $1.5 \mathrm{~kg} / \mathrm{year}$ between consecutive weight assessments were used to define mild and strong weight cycling. These classifications were chosen based on a publication from NHS II [5].

\section{Analyses on a priori defined weight patterns and diabetes} risk The association between weight patterns (weight cycling, stable weight, weight loss and weight gain) and time to type 2 diabetes occurrence was assessed using Cox proportional hazards regression, with age as the underlying timescale. Since participants were followed from the age at the last weight assessment, the data were left-truncated and study participants that were lost to follow-up or died during the follow-up period were censored. Validity of the proportional hazards assumption was tested using Schoenfeld residuals [12]. Multivariable Cox regression analyses were stratified by age (i.e. strata of 5 year integers of baseline age) and adjusted for sex and the following baseline variables: BMI, study centre, physical activity according to the Cambridge physical activity index (active, moderately active, moderately inactive, inactive), alcohol intake (continuous), smoking status (never, former, current) and educational level (low: primary school or no school graduation; medium: secondary, technical or professional school graduation; high: longer education including university degree) [13]. Physical activity and educational level were modelled as ordinal variables. Linearity of the relationship between the hazard of type 2 diabetes and either BMI or alcohol consumption was explored using restricted cubic splines. An exponential relationship between BMI and the hazard of type 2 diabetes was observed; therefore, BMI values were log-transformed. Regarding alcohol consumption, it was found that intake levels of 0-28 g/ day were associated with a lower risk of type 2 diabetes, intakes of $28-80 \mathrm{~g} /$ day were associated with a moderately higher risk of type 2 diabetes and intakes of $>80 \mathrm{~g} /$ day were associated with a clearly higher risk of type 2 diabetes. Thus, alcohol consumption was categorised by these three cut-offs. Additionally, a dichotomous variable (never/ever use of alcohol) was used as an adjustment variable in all models.

Sensitivity analyses were carried out excluding participants with a baseline BMI of $\geq 30 \mathrm{~kg} / \mathrm{m}^{2}$ from the stable weight reference group. In further sensitivity analyses, participants in the weight cycling category who had lost weight between the two last weight assessments before diagnosis were 
excluded from Cox regression analyses because this pattern of weight cycling may actually reflect unintentional weight loss due to untreated insulin resistance.

Identification of weight patterns by FPCA To account for the fact that weight cycling may occur during net weight gain or loss, FPCA [14] was used to assess the dominant modes of BMI variation from baseline to the last follow-up, as proposed by Waring et al [15]. Average BMI patterns were computed and the amount of their total variation that could be explained by each of the principal component (PC) functions was investigated. For each participant, the individual BMI trajectory was modelled as a linear combination of four quadratic Bspline basis functions using one interior knot [16].

The first PC function reflects a constant shift of the average BMI curve and accounts for $96.1 \%$ of the variation in the study population (see ESM Fig. 2). Thus, participants with high PC1 scores have a BMI above average and participants with lower PC1 scores have a BMI below average. Using standard cut-offs for BMI (normal weight: $>18.5$ and $<25 \mathrm{~kg} / \mathrm{m}^{2}$; overweight: $\geq 25$ and $<30 \mathrm{~kg} / \mathrm{m}^{2}$; obesity: $\geq 30 \mathrm{~kg} / \mathrm{m}^{2}$ ), study participants with a PC score in the deciles 1-5 were classified as having overall normal weight, participants with PC score in deciles 6-9 as overweight and participants with a PC score in the 10th decile as obese.

The second PC function reflects linear contrast between the first and second half of the BMI curve and explains $2.8 \%$ of the variation in weight patterns of the study population. A high PC score indicates weight gain, a low score indicates weight loss and a score close to the median indicates stable weight. PC scores in deciles 1-4 indicate weight loss, scores in deciles 5-6 indicate stable weight and scores in the deciles 7-10 indicate weight gain. The third and fourth PCs reflect a cyclic BMI pattern. Together, they account for $1.1 \%$ of the variation in BMI patterns. In this case, any score different from zero indicates a multiple of a cycling rate of about $1 \mathrm{~kg} / \mathrm{m}^{2}$.

\section{Analyses on weight patterns identified by FPCA and dia-} betes risk For Cox proportional hazards models, categorical variables of weight change derived from the PC scores (PC1: normal weight, overweight, obese; PC2: weight loss, stable weight, weight gain; PC3 and PC4: non-cycling, weight cycling) were used to estimate associations between BMI patterns and the hazard of type 2 diabetes, while adjusting for the same set of potential confounders as in the analyses described above, i.e. sex, initial BMI, study centre, physical activity, alcohol intake, smoking status and educational level. In addition, the identified PCs were adjusted for each other. Pairwise interactions among weight cycling, weight change and overall weight status were evaluated by adding interaction terms for the variables to a multivariable Cox regression model.

The threshold for statistical significance after Bonferroni adjustment for multiple testing was $p<0.0125(0.05 / 4)$, considering four independent tests (a priori method with three different cut-offs and the FPCA approach). Statistical analyses were carried out using SAS 9.3 (SAS Institute, Cary, NC, USA) and R version 3.1 .0 (see www.r-project.org).

\section{Results}

Descriptive results The final sample consisted of 643 incident type 2 diabetes patients and 44,420 non-diabetic individuals. Characteristics of study participants by patterns of weight change are presented in Table 1.

Median follow-up time for the analytical cohort was 2.5 years (interquartile range $=0.9$ years). Participants categorised as weight cyclers had a higher weight and waist circumference at baseline compared with participants in the weight gain and stable weight group. The weight loss group showed the highest initial weight and waist measurements overall. The prevalence of weight cycling (as defined by average annual weight changes of $1.125 \mathrm{~kg}$ ) was $13.9 \%$ in the overall cohort and higher among participants who subsequently developed type 2 diabetes $(18.8 \%)$ compared with those who did not $(13.8 \%)$. The prevalence estimates of weight cycling as identified by FPCA were $14.5 \%$ in later cases of type 2 diabetes and $10.0 \%$ in non-cases, respectively.

A priori defined weight cycling and diabetes risk Cox regression analyses showed that after adjustment for BMI at baseline and further confounders, weight gain (HR 2.08 [95\% CI 1.60, 2.70]) and weight cycling (HR 1.36 [95\% CI $1.09,1.68])$ were both significantly associated with a higher hazard of type 2 diabetes (see Table 2).

Results from Cox regression analyses obtained using the alternative a priori defined cut-offs of weight cycling ( 0.75 and $1.5 \mathrm{~kg} /$ year) are shown in Table 3 . While both mild weight cycling, as defined by the $0.75 \mathrm{~kg} /$ year criterion (HR 1.77 [95\% CI 1.45, 2.16]), and severe weight cycling, as defined by the $1.5 \mathrm{~kg} / \mathrm{year}$ criterion (HR 2.09 [95\% CI 1.63, 2.68]), were significantly associated with a higher hazard of diabetes in Model 1, these associations were attenuated after further correction for BMI at baseline $(0.75 \mathrm{~kg} / \mathrm{year}$ : HR 1.20 [95\% CI 0.98, 1.48], 1.5 kg/year: HR 1.34 [95\% CI $1.03,1.73])$. When excluding participants with a BMI of $\geq 30 \mathrm{~kg} / \mathrm{m}^{2}$ from the stable weight category in the analyses based on the $1.125 \mathrm{~kg} /$ year cut-off, associations between weight cycling, weight gain and diabetes risk remained very similar (ESM Table 1). Exclusion of participants who had lost weight between the last two weight assessments from the weight cycling category 
Table 1 Characteristics of study participants at baseline by patterns of weight change $\mathrm{e}^{\mathrm{a}, \mathrm{b}}$

\begin{tabular}{|c|c|c|c|c|}
\hline Characteristic & Stable weight & Weight loss & Weight cycling & Weight gain \\
\hline$n$ & 35,587 & 429 & 6,243 & 2,804 \\
\hline Women & $20,418(57.4)$ & $274(63.9)$ & $3,640(58.3)$ & $1,678(59.8)$ \\
\hline Age at baseline (years) & $51.0(43.0,57.0)$ & $51.0(44.0,59.0)$ & $49.0(42.0,57.0)$ & $46.0(41.0,53.0)$ \\
\hline Weight (kg) & $71.80(62.8,81.7)$ & $87.6(77.0,98.6)$ & $78.2(68.9,88.6)$ & $73.8(65.0,85.0)$ \\
\hline Waist (cm) & $85.50(76.0,94.5)$ & $98.3(89.0,107)$ & $90.5(81,99)$ & $86.0(77.3,96.0)$ \\
\hline \multicolumn{5}{|l|}{ BMI categories $\left(\mathrm{kg} / \mathrm{m}^{2}\right)$} \\
\hline Normal weight $(<25)$ & $16,908(47.5)$ & $29(6.8)$ & $1,709(27.4)$ & $1,157(41.3)$ \\
\hline Overweight $(\geq 25)$ & $14,093(39.6)$ & $147(34.3)$ & $2,861(45.8)$ & $1,129(40.3)$ \\
\hline Obese $(\geq 30)$ & $4,586(12.9)$ & $253(59.0)$ & $1,673(26.8)$ & $518(18.4)$ \\
\hline BMI at the age of 18 years $\left(\mathrm{kg} / \mathrm{m}^{2}\right)$ & $20.6(19.3,21.6)$ & $22.15(18.9,23.5)$ & $21.4(18.8,22.7)$ & $20.3(18.7,22.5)$ \\
\hline Alcohol (g/day) & $8.9(2.9,21.6)$ & $6.8(1.7,19.4)$ & $8.0(2.3,20.8)$ & $7.3(2.0,20.0)$ \\
\hline \multicolumn{5}{|l|}{ Smoking status } \\
\hline Never & $16,919(47.5)$ & $169(39.4)$ & $2,688(43.1)$ & $1,109(39.6)$ \\
\hline Former & $11,669(32.8)$ & $166(38.7)$ & $2,188(35.0)$ & $845(30.1)$ \\
\hline Smoker & 6,999 (19.7) & $94(21.9)$ & $1,367(21.9)$ & $850(30.3)$ \\
\hline \multicolumn{5}{|l|}{ Physical activity $^{c}$} \\
\hline Inactive & $5,624(15.8)$ & $72(16.8)$ & $1,176(18.8)$ & $548(19.5)$ \\
\hline Moderately inactive & $12,904(36.3)$ & $169(39.4)$ & $2,288(36.6)$ & $1,048(37.4)$ \\
\hline Moderately active & $9,486(26.7)$ & $119(27.7)$ & $1,621(26.0)$ & $681(24.3)$ \\
\hline Active & $7,573(21.3)$ & $69(16.1)$ & $1,158(18.5)$ & $527(18.8)$ \\
\hline \multicolumn{5}{|l|}{ Education } \\
\hline None & $213(0.6)$ & $2(0.5)$ & $38(0.6)$ & $10(0.4)$ \\
\hline Primary school completed & $8,405(23.6)$ & $111(25.9)$ & $1,389(22.2)$ & $592(21.1)$ \\
\hline Technical/professional school & $12,408(34.9)$ & $164(38.2)$ & $2,306(36.9)$ & $1,118(39.9)$ \\
\hline Secondary school & $2,435(6.8)$ & $31(7.2)$ & $400(6.4)$ & $211(7.5)$ \\
\hline Longer education (including degree) & $12,126(34.1)$ & $121(28.2)$ & $2,110(33.9)$ & $873(31.1)$ \\
\hline Incident diabetes & $437(1.2)$ & $8(1.9)$ & $121(1.9)$ & $77(2.7)$ \\
\hline
\end{tabular}

${ }^{\text {a }}$ Presented values are $n(\%)$. Median (25th, 75th percentiles) are shown for age, weight and waist circumference at baseline, BMI at age 18 years and alcohol intake

${ }^{\mathrm{b}}$ Based on an average annual weight change of $1.125 \mathrm{~kg} /$ year between two weight assessments; individuals in the weight cycling category showed at least one period of weight loss of $\geq 1.125 \mathrm{~kg} /$ year followed by subsequent regain or vice versa

${ }^{\mathrm{c}}$ According to the Cambridge Physical Activity Index

Table 2 HR for type 2 diabetes according to a priori defined patterns of weight change ${ }^{\mathrm{a}}$

\begin{tabular}{|c|c|c|c|c|c|c|}
\hline \multirow[t]{2}{*}{ Diabetes } & \multirow[t]{2}{*}{ Cases } & \multirow[t]{2}{*}{ Non-cases } & \multicolumn{2}{|l|}{ Model $1^{\mathrm{b}}$} & \multicolumn{2}{|l|}{ Model $2^{\mathrm{c}}$} \\
\hline & & & HR $(95 \%$ CI $)$ & $p$ & HR $(95 \%$ CI) & $p$ \\
\hline Stable weight & 437 & 35,150 & 1.0 (Reference) & & 1.0 (Reference) & \\
\hline Weight loss & 8 & 421 & $1.53(0.75,3.09)$ & 0.24 & $0.47(0.22,1.01)$ & 0.05 \\
\hline Weight gain & 77 & 2,727 & $2.87(2.23,3.69)$ & $<0.0001$ & $2.08(1.60,2.70)$ & $<0.0001$ \\
\hline Weight cycling & 121 & 6,122 & $2.03(1.65,2.50)$ & $<0.0001$ & $1.36(1.09,1.68)$ & 0.006 \\
\hline
\end{tabular}

${ }^{a}$ Individuals in the weight cycling category showed at least one period of weight loss of $\geq 1.125 \mathrm{~kg} /$ year followed by subsequent weight regain or vice versa

${ }^{\mathrm{b}}$ Adjusted for sex, centre, physical activity (active, moderately active, moderately inactive, inactive), smoking status (never, former, current), alcohol intake (continuous; g/day) and education (low: primary school or no school graduation; medium: secondary, technical or professional school completed; high: longer education including university degree) at baseline

${ }^{\mathrm{c}}$ As for Model 1, with additional adjustment for BMI at baseline 
Table 3 HRs for type 2 diabetes according to a priori defined patterns of weight change using alternative cut-offs

\begin{tabular}{|c|c|c|c|c|c|c|}
\hline \multirow[t]{2}{*}{ Weight change } & \multirow[t]{2}{*}{ Cases } & \multirow[t]{2}{*}{ Non-cases } & \multicolumn{2}{|l|}{ Model $1^{\mathrm{a}}$} & \multicolumn{2}{|l|}{ Model $2^{\mathrm{b}}$} \\
\hline & & & $\operatorname{HR}(95 \% \mathrm{CI})$ & $p$ & HR (95\% CI) & $p$ \\
\hline \multicolumn{7}{|l|}{$0.75 \mathrm{~kg} /$ year } \\
\hline Stable weight & 327 & 27,744 & 1.0 (Reference) & & 1.0 (Reference) & \\
\hline Weight loss & 17 & 905 & $1.52(0.93,2.49)$ & 0.09 & $0.57(0.34,0.96)$ & 0.04 \\
\hline Weight gain & 135 & 6,168 & $2.15(1.74,2.66)$ & $<0.0001$ & $1.73(1.39,2.14)$ & $<0.0001$ \\
\hline Weight cycling & 164 & 9,603 & $1.77(1.45,2.16)$ & $<0.0001$ & $1.20(0.98,1.48)$ & 0.08 \\
\hline \multicolumn{7}{|l|}{$1.5 \mathrm{~kg} /$ year } \\
\hline Stable weight & 517 & 39,430 & 1.0 (Reference) & & 1.0 (Reference) & \\
\hline Weight loss & 4 & 196 & $1.58(0.59,4.28)$ & 0.36 & $0.33(0.10-1.05)$ & 0.06 \\
\hline Weight gain & 47 & 1,240 & $4.12(3.03,5.59)$ & $<0.0001$ & $2.66(1.93-3.66)$ & $<0.0001$ \\
\hline Weight cycling & 75 & 3,554 & $2.09(1.63,2.68)$ & $<0.0001$ & $1.34(1.03-1.73)$ & 0.03 \\
\hline
\end{tabular}

${ }^{a}$ Adjusted for sex, centre physical activity (active, moderately active, moderately inactive, inactive), smoking status (never, former, current), alcohol intake (continuous; g/day) and education (low: primary school or no school graduation; medium: secondary, technical or professional school completed; high: longer education including university degree) at baseline

${ }^{\mathrm{b}}$ As Model 1, additionally adjusted for BMI at baseline

did not substantially affect any of the reported associations (data not shown).

FPCA-derived weight cycling and diabetes risk After adjustment for weight change, weight cycling and confounders, overweight (HR 3.80 [95\% CI 2.63, 5.47]) and obesity (HR 9.04 [95\% CI 6.26, 13.05]) were significantly associated with a higher hazard of type 2 diabetes (see Table 4). Weight gain was not significantly associated with the hazard of type 2 diabetes after full adjustment (HR 1.20 [95\% CI 0.95, 1.51]). While a significant positive association was observed for weight cycling (PC3 and PC4) when only adjusting for standard covariates (HR 1.79 [95\% CI 1.34, 2.38]), this association was no longer significant after further adjustment for overall weight status (PC1) and weight change (PC2; HR 1.19 [95\% CI 0.89, 1.60]).

A significant interaction between overall weight status and weight cycling in relation to the hazard of type 2 diabetes was observed $\left(p_{\text {interaction }}=0.02\right)$. However, subgroup analyses did not reveal significant associations between weight cycling and the hazard of type 2 diabetes in overweight and obese (HR 2.20 [95\% CI $0.70,6.60]$ ) or in normal weight individuals (HR could not be estimated because of the small stratum size). There was an interaction between weight cycling and weight change in relation to diabetes risk $\left(p_{\text {interaction }}=0.02\right)$. Stratification by weight change showed that within the subgroup of net weight gainers, weight cycling was related to a significantly higher hazard of type 2 diabetes compared with non-cycling (HR 1.68 [95\% CI 1.14, 2.48], see Fig. 2), while no significant effect of weight cycling was detected in either the weight loss or the stable weight group.

\section{Discussion}

In the EPIC-Germany cohort, a priori defined patterns of weight cycling were associated with a higher risk of type 2 diabetes after adjustment for baseline BMI. In contrast, the pattern of weight cycling identified by the FPCA showed no significant association with the hazard of type 2 diabetes in the overall study population after adjustment for overall weight status and net weight change. The latter finding may argue against genuine effects of weight cycling in the development of type 2 diabetes. However, in the subgroup of participants characterised by net weight gain according to the FPCA, FPCA-derived weight cycling was associated with a significantly increased risk of type 2 diabetes, which may indicate adverse effects of weight cycling during phases of weight gain but not weight stability.

The present findings of significant direct associations between different categories of a priori defined weight cycling and the hazard of type 2 diabetes based on average weight changes of $0.75,1.125$ and $1.5 \mathrm{~kg} /$ year are in agreement with results from three previous studies: the DPP [8], IWS [3] and TOPS studies [4], while no associations between weight cycling and the hazard of type 2 diabetes were observed in NHS II [5]. However, it should be noted that various definitions of weight cycling were used in these studies and none of the previous classifications could be applied here in exactly the same way because of differences in the number and frequency of weight assessments during follow-up. For example, weight changes of $\geq 4.5$ and $\geq 9 \mathrm{~kg}$ over 6 years were used to define mild and severe weight cycling, respectively, in NHS II. Although the same average annual weight changes (i.e. 0.75 and $1.5 \mathrm{~kg}$ ) underlie the definitions of weight cycling in the 
Table 4 HRs for type 2 diabetes according to patterns of weight change identified by principal component analysis

\begin{tabular}{|c|c|c|c|c|c|c|}
\hline \multirow[t]{2}{*}{ Weight change pattern } & \multirow[t]{2}{*}{ Cases } & \multirow[t]{2}{*}{ Non-cases } & \multicolumn{2}{|l|}{ Models $^{\mathrm{a}}$} & \multicolumn{2}{|l|}{ Adjusted model $^{\mathrm{b}}$} \\
\hline & & & $\mathrm{HR}(95 \% \mathrm{CI})$ & $p$ & $\mathrm{HR}(95 \% \mathrm{CI})$ & $p$ \\
\hline \multicolumn{7}{|l|}{ Stable weight (PC 1) } \\
\hline Normal weight & 39 & 14,098 & 1.0 (Reference) & & 1.0 (Reference) & \\
\hline Overweight & 158 & 13,942 & $3.88(2.69,5.58)$ & $<0.0001$ & $3.80(2.63,5.47)$ & $<0.0001$ \\
\hline Obese & 202 & 6,831 & $9.35(6.52,13.42)$ & $<0.0001$ & $9.04(6.26,13.05)$ & $<0.0001$ \\
\hline \multicolumn{7}{|l|}{ Weight change (PC 2) } \\
\hline Stable weight & 149 & 15,724 & 1.0 (Reference) & & 1.0 (Reference) & \\
\hline Weight loss & 81 & 6,972 & $1.09(0.83,1.43)$ & 0.5 & $0.83(0.63,1.10)$ & 0.19 \\
\hline Weight gain & 169 & 12,175 & $1.51(1.20,1.89)$ & 0.0004 & $1.20(0.95,1.51)$ & 0.12 \\
\hline \multicolumn{7}{|c|}{ Weight cycling (PCs 3 and 4) } \\
\hline Non-cycling & 341 & 31,389 & (Reference) & & 1.0 (Reference) & \\
\hline Weight cycling & 58 & 3,482 & $1.79(1.34,2.38)$ & $<0.0001$ & $1.19(0.89,1.60)$ & 0.25 \\
\hline
\end{tabular}

${ }^{a}$ Adjusted for sex, centre physical activity (active, moderately active, moderately inactive, inactive), smoking status (never, former, current), alcohol intake (continuous; g/day) and education (low: primary school or no school graduation; medium: secondary, technical or professional school completed; high: longer education including university degree) at baseline

${ }^{\mathrm{b}}$ This model was adjusted for the same variables as the models in the first column of the table, and additionally for all other PCs (categorical variables)

present study, the average interval between the two weight assessments was shorter in EPIC-Germany (2.3 years).

As settling on a common definition of weight cycling in epidemiological studies is difficult because of differences in study design, mostly with respect to length of follow-up, an FPCA-based approach to define weight cycling was proposed in a previous publication by the FHS [6]. As in the FHS [6], the present study did not reveal any overall associations between weight cycling pattern and type 2 diabetes risk after adjustment for PCs reflecting stable weight patterns and constant weight change. Nevertheless, FPCA-derived weight cycling was indeed associated with a higher hazard of type 2 diabetes in net weight gainers. Therefore, the present findings suggest that weight cycling leads to adverse effects during substantial net weight gain. Certainly, this interpretation is not consistent with the observation of weight cycling induced visceral fat accumulation in normal weight, rather than overweight and obese individuals and requires confirmation in other studies [17].

Weight loss $0.82(0.44,1.55)$

81 cases (13 cyclers, 68 non-cyclers)

Stable weight $0.93(0.46,1.86)$

149 cases ( 9 cyclers, 140 non-cyclers)

Weight gain $1.68(1.14,2.48)$

169 cases ( 36 cyclers, 133 non-cyclers)

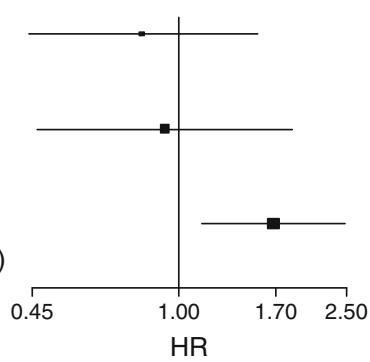

Fig. 2 Associations between weight cycling and the HR for type 2 diabetes across FPCA-derived categories of weight change
There are specific strengths and limitations to statistical formalisations of weight development over time. A priori definitions of weight patterns are based on mutually exclusive categories that classify individuals as either cyclers or noncyclers (weight gain, stable weight, weight loss). The complexity of weight development is not reflected because a person may experience several of these patterns simultaneously. Most importantly, concurrent patterns (e.g. weight cycling during net weight gain) cannot be sufficiently accounted for. The classification of individuals demonstrating concurrent patterns is arbitrary because they fall into more than one category. Conversely, while the classification of individuals into weight pattern categories using FPCA is designed to capture the full complexity of weight development, the reliability of the method is dependent on the number of follow-up measurements. In the present study, only four weight measurements per participant were available, which is a main limitation. However, while analyses based on more repeated assessments would more accurately capture weight fluctuations over time, tight monitoring of weight over several years may not be feasible in large cohort studies such as ours with a meaningful follow-up duration.

Further limitations of the analyses on weight cycling and diabetes from EPIC-Germany need to be considered. One is that data on intentionality of weight loss were not available. However, participants with major chronic diseases (i.e. cancer, myocardial infarction and stroke) were excluded from the present analyses, and this may have limited confounding due to unintentional weight loss. Another clear limitation of the present study is that weight during follow-up was self-reported. However, as weight was possibly under-reported rather 
than over-reported, at least in women [18], it could be speculated that this may even have led to underestimation of the statistical effects of weight cycling. Furthermore, validated equations were used to correct for self-reported weight. Although subgroup analyses from EPIC-Germany suggest the health effects of weight cycling during weight gain, it should be acknowledged that the sample size in these analyses was limited and that biological mechanisms underlying possible adverse effects of weight cycling are far from clear [19]. In addition, observations from weight loss trials do not consistently indicate that abdominal [20] or visceral fat accumulation [21] is induced by weight cycling.

Taken together, the present findings suggest that, unlike obesity, weight cycling may not be a strong independent risk factor for type 2 diabetes in the overall adult population, when concurrent patterns of weight change and overall weight status are accounted for. At the same time, subgroup findings suggested that weight cycling during net weight gain may pose a stronger risk of diabetes compared with non-cycling. The latter observation should be considered a starting point for future studies and requires cautious interpretation until confirmed by others.

Funding This work was supported by Kompetenznetz Adipositas (Competence Network Obesity), funded by the German Federal Ministry of Education and Research (grant no. 01GI1121B).

Duality of interest The authors declare that there is no duality of interest associated with this manuscript.

Contribution statement $\mathrm{RK}$ and $\mathrm{HB}$ designed the study and acquired data. JN-A and MB carried out the statistical analyses. JN-A, MB, AH, VAK, UB, AS, RK, MBS, HB and TK interpreted data. JN-A, MB and TK drafted the paper. All authors critically revised the manuscript for important intellectual content and approved the final version. TK is responsible for the integrity of the work as a whole.

\section{References}

1. Hu FB (2011) Globalization of diabetes: the role of diet, lifestyle, and genes. Diabetes Care 34:1249-1257

2. Kodama S, Horikawa C, Fujihara K et al (2014) Quantitative relationship between body weight gain in adulthood and incident type 2 diabetes: a meta-analysis. Obes Rev 15:202-214

3. French SA, Folsom AR, Jeffery RW, Zheng W, Mink PJ, Baxter JE (1997) Weight variability and incident disease in older women: the Iowa Women's Health Study. Int J Obes Relat Metab Disord 21:217-223

4. Morris RD, Rimm AA (1992) Long-term weight fluctuation and non-insulin-dependent diabetes mellitus in white women. Ann Epidemiol 2:657-664
5. Field AE, Manson JE, Laird N, Williamson DF, Willett WC, Colditz GA (2004) Weight cycling and the risk of developing type 2 diabetes among adult women in the United States. Obes Res $12: 267-274$

6. Waring ME, Eaton CB, Lasater TM, Lapane KL (2010) Incident diabetes in relation to weight patterns during middle age. Am J Epidemiol 171:550-556

7. Mehta T, Smith DL Jr, Muhammad J, Casazza K (2014) Impact of weight cycling on risk of morbidity and mortality. Obes Rev $15: 870-881$

8. Delahanty LM, Pan Q, Jablonski KA et al (2014) Effects of weight loss, weight cycling, and weight loss maintenance on diabetes incidence and change in cardiometabolic traits in the diabetes prevention program. Diabetes Care 37:2738-2745

9. Boeing H, Wahrendorf J, Becker N (1999) EPIC-Germany-a source for studies into diet and risk of chronic diseases. Ann Nutr Metab 43:195-204

10. Haftenberger M, Lahmann PH, Panico S et al (2002) Overweight, obesity and fat distribution in 50-to 64 year-old participants in the European Prospective Investigation into Cancer and Nutrition (EPIC). Public Health Nutr 5:1147-1162

11. Spencer EA, Appleby PN, Davey GK, Key TJ (2002) Validity of self-reported height and weight in 4808 EPIC-Oxford participants. Public Health Nutr 5:561-565

12. Grambsch PM, Therneau TM (1994) Proportional hazards tests and diagnostics based on weighted residuals. Biometrika 81:515-526

13. Wareham NJ, Jakes RW, Rennie KL et al (2003) Validity and repeatability of a simple index derived from the short physical activity questionnaire used in the European Prospective Investigation into Cancer and Nutrition (EPIC) study. Public Health Nutr 6:407-413

14. Ramsay JO, Hooker G, Graves S (2009) Exploring variation: functional principal and canonical components analysis. In: Gentleman R, Hornik K, Parmigiani G (eds) Functional data analysis with R and MATLAB. Springer, London, pp 99-115

15. Waring ME, Eaton CB, Lasater TM, Lapane KL (2010) Correlates of weight patterns during middle age characterized by functional principal components analysis. Ann Epidemiol 20:201-209

16. Ramsay JO, Hooker G, Graves S (2009) Smoothing: computing curves from noisy data. In: Gentleman R, Hornik K, Parmigiani G (eds) Functional data analysis with R and MATLAB. Springer, London, pp 29-38

17. Bosy-Westphal A, Muller MJ (2014) Measuring the impact of weight cycling on body composition: a methodological challenge. Curr Opin Clin Nutr Metab Care 17:396-400

18. Merrill RM, Richardson JS (2009) Validity of self-reported height, weight, and body mass index: findings from the National Health and Nutrition Examination Survey, 2001-2006. Prev Chronic Dis 6:A121

19. Bosy-Westphal A, Schautz B, Lagerpusch M et al (2013) Effect of weight loss and regain on adipose tissue distribution, composition of lean mass and resting energy expenditure in young overweight and obese adults. Int J Obes 37:1371-1377

20. Cereda E, Malavazos AE, Caccialanza R, Rondanelli M, Fatati G, Barichella M (2011) Weight cycling is associated with body weight excess and abdominal fat accumulation: a cross-sectional study. Clin Nutr 30:718-723

21. van der Kooy K, Leenen R, Seidell JC, Deurenberg P, Hautvast JG (1993) Effect of a weight cycle on visceral fat accumulation. Am J Clin Nutr 58:853-857 